\title{
ORDER IDEAL LIMIT POINTS AND A GENERALIZED BOLZANO-THEOREM
}

\author{
BABLU BiswaS AND D. K. GANGUly
}

Abstract. In this paper we extend the concept of I-limit points and I-cluster points in a linearly ordered metric additive system and study the notion of $O I$-limit points and $O I$-cluster points and also establish a generalization of Bolzano-Weierstrass theorem connected to a bounded sequence.

Mathematics subject classification (2010): Primary 40A05; Secondary 46B42.

Keywords and phrases: Additive system, order convergence, natural density, ideal, I-convergence, OIconvergence.

\section{REFERENCES}

[1] P. Kostyrko, T. Salat, W. Wilczynski, I-convergence, Real Analysis Exchange 26, 2 (2000), 669-688.

[2] P. Kostyrko, M. Macaj, T. Salat, M. Sleziak, I-convergence and External I-limit Points, Math. Slovaca 55 (2005), 443-464.

[3] K. Dems, On I-Cauchy sequences, Real Analysis Exchange 30 (2004), 123-128.

[4] F. GeZer, S. KARAKUS, I and $I^{*}$ convergent function sequences, Math. Commun. 10 (2005), 71-80.

[5] B. C. TRIPATHY, On I-convergent double sequence, Soochow J. of Math. 31, 4 (2005), 549-560.

[6] B. C. TRIPATHY, B. HAZARIKA, I-convergent sequence spaces associated with multiplier sequence spaces, Mathematical Inequalities and Applications 11, 3 (2008), 543-548.

[7] B. C. TRipathy, S. Mahanta, On I-acceleration convergence of sequences, Journal of the Franklin Institute 347 (2010), 591-598.

[8] B. C. TRIPATHY, M. Sen, S. NATH, I-convergence in probabilistic n-normed space, Soft Comput. 16 (2012), 1021-1027, DOI 10.1007/s00500-011-0799-8.

[9] B. C. TRipathy, A. J. DutTa, On I-acceleration convergence of sequences of fuzzy real numbers, Math. Modell. Analysis 17, 4 (2012), 549-557.

[10] B. C. TRIPATHY, B. SARMA, On I-convergent double sequences of fuzzy real numbers, Kyungpook Math. Journal 52, 2 (2012), 189-200.

[11] T. Salat, B. C. Tripathy, M. Ziman, On I-convergence field, Italian J. of Pure Appl. Math. 17 (2005), 45-54.

[12] J.A. FrIDY, Statistical limit points, Proc. Amer. Math. Soc. 118 (1993), 1187-1192.

[13] J. A. Fridy, C. Orhan, Statistical limit superior and limit inferior, Proc. Amer. Math. Soc. 125 (1997), 3625-3631.

[14] H. FAST, Sur la convergence statistique, Colloquium Matyhematicum 2 (1959), 241-244.

[15] I. J. SCHOENBERG, The integrablity of certain functions and related summability methods, The American mathematical Monthly 66, 5 (1959), 361-375.

[16] H. Steinhaus, Sur la convergence ordinaire et la convergence asymptotique, Colloquium Matyhematicum 2 (1951), 73-74.

[17] H.I.MilleR, A measure theoritical subsequence characterization of statistical convergence, Transactions of the American Mathematical Society 374, 5 (1995), 1811-1819.

[18] J.A. FRIDY, On statistical convergence, Analysis 5, 4 (1985), 301-313.

[19] T. SALAT, On statistical convergence of real numbers, Math. Slovaca. 30, 2 (1980), 139-150.

[20] L. R. Wilcox And M. F. Smiley, Metric Lattices, Annals of Mathematics 40, 2 (April 1939), 309-327. 
[21] G. BIR Khoff, Lattice Theory, Amer. Math. Soc. Colloq. Pubi. vol. XXV (First Edition), New York, 1940.

[22] O. FrinK, Topology in lattices, Trans. Amer. Math. Soc. 51 (1942), 569-582.

[23] Charalambos D. Aliprantis, Kim C. Border, Infinite Dimensional Analysis, Springer Berlin Heidelberg (Third Edition), New York, 2006.

[24] D. K. Ganguly, B. Biswas, Order ideal convergence convergence in a metric space, Communicated.

[25] I. Niven, H. S. Zuckerman, An Introduction to the Theory of Numbers, John Wiley \& Sons, New York, USA, 4-th edition, Chapter-11, 1980.

[26] B. C. TRIPAThY, B. HAZARIKA, On I-monotonic and I-convergent sequences, Kyungpook Math. Journal 51, 2 (2011), 233-239.

[27] T. Salat, B. C. Tripathy, M. Ziman, On some properties of I-convergence, Tatra Mt. Math. Publ. 28 (2004), 279-286. 